Fecha de recepción: agosto 2008

Fecha de aceptación: marzo 2009

Versión final: diciembre 2010

\section{La construcción del espacio académico: ciencia y diversidad}

Irene Scaletzky ${ }^{(*)}$

\begin{abstract}
Resumen: La problemática de la diversidad cultural se ha transformado en uno de los ejes del proceso académico y pedagógico, tanto en lo concerniente a la producción de saberes capaces de dar cuenta de dicho fenómeno como de estrategias de intervención pedagógica que nos permitan vehiculizar los procesos educativos con el éxito esperado.

En este contexto de complejización y multiplicidad simbólica de los espacios áulicos, se representan inconmensurables unidades de sentido que potencian "lo conocido" y nos desafían en la búsqueda continua de nuevas propuestas y estrategias de acción que, en decir de García Canclini (2004) nos orienten respecto de "dónde encontrar la teoría que organice las nuevas diversidades".

La Globalización mundial se ha constituido en un presente organizador de procesos sociales de sensible movilidad y, al mismo tiempo, en productor de perspectivas relacionales que en si mismas se constituyen en un desafío cognitivo en lo que a educación se refiere.

El presente trabajo se propone reflexionar sobre estos procesos sociales y educativos, asumiendo que el rol del espacio académico y por ende del pensamiento científico, resultan definitorios en la construcción de sociedades interculturales en las que la mencionada "problemática de la diversidad cultural" encuentre formas de potenciarse en nuevos conocimientos motivadores del pensamiento democrático.
\end{abstract}

Palabras claves: diversidad cultural - espacio académico - intervención pedagógica - pensamiento científico.

[Resúmenes en inglés y portugués y currículum en las páginas 106-107]

Los códigos fundamentales de una cultura -los que rigen su lenguaje, sus esquemas perceptivos, sus cambios, sus técnicas, sus valores, la jerarquía de sus prácticas- fijan de antemano para cada hombre los órdenes empíricos con los cuales tendrá algo que ver y dentro de los que se reconocerá. En el otro extremo del pensamiento, las teorías científicas o las interpretaciones de los filósofos explican por qué existe un orden en general, a qué ley general obedece, qué principio puede dar cuenta de él, por qué razón se establece este orden y no aquel otro. (Foucault, Michel, 1993: p 5-6).

Desafiando los límites del paradigma geocéntrico, filósofos, astrónomos, físicos, artistas y otras gentes, establecieron un nuevo reinado: el poder de la razón y la percepción del mundo organizada a través del pensamiento científico; con ello se abrieron las puertas a un nuevo orden social. El hombre en su carácter de individuo ungido de raciocinio intelectual se constituyó en el eje de unas articulaciones sociales y políticas que dominaron la escena mundial. Con el comienzo del Siglo XXI la humanidad experimenta nuevamente uno de esos momentos trascendentes de su devenir: 
la globalización planetaria ha producido la ruptura de ese paradigma racional y ha expuesto unas realidades tan diversas a lo anteriormente conocido que como mínimo, nos enfrenta a la ardua tarea de intentar comprenderlas.

En este panorama de cambios, lo primero que surge claramente es una gran confusión: ¿qué alcances cotidianos tiene la ruptura de un paradigma cognitivo y social? Lo que se ha quebrado, ¿cómo se expresa? ¿Qué se hace con ello? Podríamos transcribir una importante cantidad de interrogantes y creemos no equivocarnos si afirmamos que, la gran mayoría de ellos estaría representando distintos aspectos de este fenómeno novedoso en el que transcurre nuestro presente. Sin embargo, a pesar de constatar cotidianamente esos cambios, no podemos dejar de reconocer, con igual certeza, que los mismos no siempre constituyen rupturas drásticas sino más bien movimientos de adaptación, en sentidos diversos, que reflejan nuevas configuraciones sociales y simbólicas, en la mayoría de los casos representados por las nuevas generaciones.

Si bien cambios y rupturas caracterizan a la Globalización en sí, el propósito específico del presente trabajo está centrado en el análisis de su impacto en el ámbito de las nuevas configuraciones sociales que se expresan en el espacio académico, puntualmente en el aula universitaria. Consideramos que dicho ámbito, en el que se ponen en juego todas esas características del proceso de cambio social representadas no sólo por la diversa constitución del alumnado sino también por los roles asumidos por las instituciones, refleja una de relaciones más productivas en cuanto a las posibilidades de establecer nuevos diálogos entre la ciencia y la mencionada diversidad cultural.

\section{La "invención” de las diferencias}

Lo primero que se nos plantea es esta suerte de "nueva preocupación" por la existencia de universos culturales diferenciales que de modo repentino son llevados a una convivencia desnaturalizada. Los años ochenta vieron el estallido de una problemática socio-política caracterizada por el hundimiento de las experiencias socialistas y la salida de los modelos de capitalismo benefactor. Ambos conflictos provocaron un impacto devastador en las sociedades europeas que rápidamente se extendió hacia el resto del planeta. Lo que sobrevino fue un largo período de crisis que a su vez dio paso a unas configuraciones económicas, sociales y políticas que en varios aspectos han ido marcando nuevos senderos de acción y nuevas articulaciones socio-culturales de profunda repercusión en el ámbito de lo político y lo económico. Sobre estos fenómenos han proliferado diversas propuestas teóricas provenientes del campo de la filosofía, la política, la sociología y el resto de las ciencias sociales. La mayoría de ellas han hecho hincapié en las rupturas de los modelos representativos de la segunda mitad del siglo XX he incluso han abordado desde distintos ángulos el surgimiento de un nuevo paradigma que se condiga con el nuevo mercado global.

Ahora bien, lo que aparece como novedad es el golpe de gracia que esta crisis ha provocado sobre el andamiaje de los, aparentemente sólidos, estados nacionales y sus homogéneas sociedades civiles. Podríamos hacer aquí un paréntesis en nuestro trabajo y dedicarnos a analizar con mayor profundidad los significados de estas construcciones simbólicas de lo social; podríamos incluso realizar un recorrido interdisciplinar de las formas en las que se cristalizó dicha representación en el imaginario social pretendiendo dilucidar hasta qué punto, por ejemplo los estados de América Latina, fueron verdaderas organizaciones nacionales según el mandato teórico europeo heredado del siglo XIX. Asumiendo la importancia de dichos recorridos investigativos como punto central en la búsqueda por comprender los alcances de unas realidades muchas veces confusas y desconcertantes, 
creemos que las mismas exceden el marco de la propuesta de trabajo y por ello sólo nos remitiremos a abordar los aspectos que a nuestro entender inciden en la conformación de la misma.

Esa "nueva preocupación" requiere también de un proceso de contextualización conceptual. "Las palabras aparecen para responder a ciertos interrogantes, a ciertos problemas que se plantean en períodos históricos determinados y en contextos sociales y políticos específicos" (Cuche, 2004: 9). Para Miguel León-Portilla es la antropología americanista la ciencia responsable de "inventar" el concepto de "diferencia" tanto social como cultural, constituyéndolo en su objeto de estudio. "De un modo o de otro, la invención de la diferencia se da acompañada de procesos cognoscitivos dirigidos a la estructuración de un discurso ordenador de sus elementos y rasgos. Y, en paralelo con tales procesos cognoscitivos y de expresión discursiva de invención y ordenamiento, las indagaciones en torno a la diferencia abren la puerta a posibles usos, con variados propósitos, de lo que se considera saber de lo peculiar del otro" (2001: 397).

La temática de la otredad ha sido abordada por las ciencias sociales a partir de las distintas escuelas de interpretación. Además de los diferentes planos en los que puede categorizarse dicha problemática, el filósofo del lenguaje Tzvetan Todorov plantea que el "Otro" puede concebirse como un grupo social concreto dentro de la sociedad o exterior a ella; en cualquier caso, ese "otro" se constituye en un sujeto ajeno al "nosotros" (1987:13). En este mismo sentido, Susana Devalle observa que hay que considerar la dimensión del observador entendiendo que la invención de un otro genérico se origina en una sociedad determinada en la cual también se construyen representaciones de un nosotros genérico; si a esta categoría "no se le atribuye especificidad histórica, cultural ni social resulta de escaso o nulo valor conceptual" (1995: 23). La otredad, originalmente circunscripta a las llamadas "sociedades primitivas", fue transformándose en un concepto aplicable a las sociedades escindidas del "nosotros nacional", es decir, a aquellos grupos, etnias o naciones que si bien pertenecían a la jurisdicción de los Estados-nación, se diferenciaban por innumerables elementos. Esta transformación del uso conceptual de la otredad tuvo en América Latina uno de sus epicentros más destacados durante los años setenta y permitió resignificar el uso del concepto "diferencia" como catalizador de problemáticas étnicas, sociales y políticas que dejaban entrever la necesidad de abrir el debate respecto de las sociedades nacionales.

\section{El impacto de la globalización en la producción de diversidad}

El proceso social que se ha ido desarrollando desde fines de la década de los setenta, cuya profundización hemos observado entre los años ochenta y noventa del siglo pasado, se ha caracterizado por una impresionante fragmentación de las organizaciones políticas y económicas, sociales y culturales, conocidas hasta la fecha. "La historia de los veinte años que siguieron a 1973 es la historia de un mundo que perdió su rumbo y se deslizó hacia la inestabilidad y la crisis". La revolución tecnológica, los cambios en los patrones de producción, la expansión de los mercados centrales conjuntamente con el achicamiento de las economías tercermundistas, el aumento del desempleo y las desigualdades de oportunidades, produjeron el debilitamiento y posterior caída de los estados nacionales lo que provocó un deterioro de sus prerrogativas y la fragmentación de un mundo que, hasta esa fecha, se había acostumbrado a un equilibrio social, político y económico cuyo garante era el propio estado (Hobsbawm, 1995: 403-431). En este marco de rupturas a nivel planetario, la globalización se fue consolidando como el nuevo contexto de inserción social y político en el que, como mencionamos anteriormente, alcanzaron nuevos significados los conceptos de otredad y diversidad. 
La temática de las diferencias se ha transformado en objeto de estudio y también en cuestión de estado. Para García Canclini:

Es un asunto teórico y es un dilema clave en las políticas sociales y culturales. No solo cómo reconocer las diferencias, cómo corregir las desigualdades y cómo conectar a las mayorías a las redes globalizadas. Para definir cada uno de estos tres términos es necesario pensar los modos en los que se complementan y se desencuentran. Ninguna de estas cuestiones tiene el formato de hace 30 años. Cambiaron desde que la globalización tecnológica interconecta simultáneamente casi todo el planeta y crea nuevas diferencias y desigualdades (2004: 14).

¿Cuál es el alcance de semejante impacto?, es decir ¿hasta qué punto el proceso de integración planetaria puede constituirse en generador de diversidad? Lo primero que se presenta es la percepción de una contradicción, porque si bien ya hicimos referencia a las rupturas y fragmentaciones que se fueron sucediendo en las últimas décadas causando la crisis del modelo de estado nacional conocido, la globalización aparece por un lado, como un nuevo fenómeno de integración en el que, al mismo tiempo, se sostienen discursos y prácticas que tienden a homogeneizar las hábitos de consumo y las ideas y, por el otro, se profundizan las tendencias divergentes en cuanto a los enfrentamientos entre grupos culturales e incluso a la producción de discursos valorativos de esas diferencias. Defensores y detractores del proceso de globalización pueden presentar argumentos a su favor que reflejen distintos aspectos de la situación mundial e incluso, pretender presagiar el rumbo que dicha problemática adquirirá en los próximos años considerando que la misma se presenta, en uno u otro sentido, predestinada. Sin embargo, no creemos que esto pueda realizarse fehacientemente; por el contrario, estamos casi convencidos que el devenir de las sociedades se encuentra en permanente movilidad, cambio y construcción y ello resulta del continuo contacto, de la constante relación e intercomunicación entre los diferentes individuos, grupos, culturas, etnias, religiones, sociedades, etc.

La problemática de la diversidad no es otra que la problemática de las relaciones sociales asimétricas, producto de unos ordenamientos políticos específicos; resulta de la imposibilidad de establecer canales de interrelación entre los diversos "nosotros genéricos" y "los otros genéricos" consecuentes. La Globalización ha producido un mapa de conexiones interplanetarias que desafía nuestro universo de sentido y nos posibilita la apertura de nuevos ámbitos de análisis para el desarrollo de nuevos saberes sobre dicha problemática.

Planteado entonces este marco contextual respecto de la "diversidad", nos centraremos ahora en el análisis de la educación y su rol social, intentaremos establecer qué lugar ocupa la universidad así como también delinear algunas perspectivas de trabajo en relación con la construcción de un espacio académico en el que se articulen conexiones entre los diversos sujetos participantes y el pensamiento científico.

\section{La educación, la universidad y el modelo socio-cultural}

La educación en nuestro país ha sido y sigue siendo centro de importantes estudios, frondosas teorías y profundos debates. A pesar de las enormes divergencias que caracterizan este campo del conocimiento, existe consenso respecto del análisis del modelo fundacional de nuestra educación que, con matices varios, ha caracterizado a los distintos países de la región: educar fue sinónimo de poblar, de civilizar, de nacionalizar y por supuesto, de la búsqueda por constituir sociedades 
nacionales en paz y progreso. Este modelo se propuso articular todos los niveles del sistema educativo alrededor de la premisa: "inclusión por homogeneización" (Dussel, 2003). Las incipientes sociedades nacionales, receptoras de importantes afluentes migratorios de amplia procedencia, contaron con un sistema educativo que priorizó la integración y asimilación de esa diversidad a través de la aplicación de un modelo teórico-práctico homogeneizante, que fuera capaz de "borrar" todas las señales de esas diferencias culturales.

Exitoso en la consecución de sus objetivos primarios, dicho modelo educativo y social entró en crisis por su imposibilidad de generar los procesos de actualización metodológica y curricular que reflejaran los requerimientos de una sociedad que a las claras, daba importantes muestras de haber iniciado cambios nodales. Los años ochenta se caracterizaron por una búsqueda desenfrenada de respuestas a los innumerables interrogantes que los mismos agentes educativos y la sociedad en su conjunto formulaban. Y, aunque el nivel universitario fue el menos "salpicado" por esta suerte de torbellino de críticas y discusiones, no tardó mucho tiempo más en abrir las puertas a los debates respecto de su propio rol educativo y social.

Que la Universidad en Argentina, en América Latina y en el mundo entero cumple un papel trascendental en la construcción social, resulta a estas alturas una obviedad. En su ya clásica investigación sobre la Universidad y el desarrollo de las profesiones, Gómez Campo y Tenti Fanfani analizan la expansión del sistema universitario a partir del concepto de "racionalización" weberiano: "La extensión del modo de vida capitalista se expresa en un proceso permanente de racionalización de todas las prácticas sociales. La búsqueda de los medios más adecuados para el logro de fines supone una expansión paralela del saber racional, saber técnico acerca de los medios" (1989: 13). De ello resulta el requerimiento de un sistema metódico de enseñanza en el que se instrumenten todos los procedimientos que permitan alcanzar la especificidad cognitiva. La sociedad transita ese proceso de complejización política, económica y cultural al mismo tiempo que construye las instituciones rectoras de la producción de saberes específicos para su propia conducción como también para la consecución de su desarrollo.

Sin embargo, es interesante observar cómo la crisis del paradigma moderno y la llegada de la globalización afectaron al nivel superior de la educación sin poner en discusión su cristalizado lugar en el proceso de construcción social. Es decir, la Universidad en nuestro país y con distintos matices en todo el mundo, sigue siendo el lugar, la institución en la que se forman los profesionales, los especialistas en los distintos saberes que la sociedad en un momento específico de su historia requiere. Y es específicamente ese rol el que dio forma a su creación y la mantiene, vaivenes más o menos, como única productora o productora casi exclusiva de dichos conocimientos.

Quizás ésta represente la primera paradoja de la educación superior, entendiendo que si la Universidad es la institución productora por excelencia del saber científico y éste como interpretante de las necesidades sociales se encuentra atravesando una de sus crisis más profunda, cabría preguntarse cómo impacta dicha crisis en el cotidiano institucional e incluso hasta dónde la misma generaría la resignificación social e histórica de su rol. Si el cambio científico-tecnológico genera a su vez una importante modificación en los parámetros del merado laboral y en las necesidades sociales, la formación de los profesionales y su práctica resultante no pueden asumirse inmóviles. Es decir que las instituciones universitarias son, al mismo tiempo, impactadas por los cambios sociales y productoras de los mismos, lo que nos lleva a pensarlas en esta relación dialéctica de cambio continuo.

El modelo clásico de la estructura del conocimiento curricular se basa en dos campos centrales: teoría y práctica, vistas como campos independientes que luego deben encontrar su interconexión 
en el ejercicio profesional. Este modelo, si se quiere, reproduce una dicotomía social entre los conocimientos generados por el pensamiento científico y los resultantes de la práctica cotidiana de vastos sectores sociales que no participan de la educación superior. Durante muchos años la división entre teoría y práctica caracterizó no sólo la formación profesional sino básicamente las formas en las que se organizaron las sociedades.

En todos los curricula debería existir este espacio de aprendizaje, que podríamos denominar "de educación para la incertidumbre". Este espacio tiende a ser más holgado en las profesiones con bajo grado de convergencia. Al mismo tiempo, su importancia aumenta en la misma medida en que tanto la evolución permanente de la conciencia social, como de la ciencia y la tecnología, convierten en no deseables u obsoletas las soluciones que en un tiempo fueron valoradas positivamente sin discusión alguna. Esta perspectiva cambia totalmente el enfoque clásico de la formación profesional, la cual ya no consiste en aprender un conocimiento codificado para reconocer-resolver ciertos problemas, sino en desarrollar capacidades más fundamentales, orientadas a manejarse en contextos complejos y en situaciones imprevistas. (Tenti Fanfani, 1989: 54).

Es en este marco en el que pretendemos profundizar nuestro análisis y nuestra propuesta ya que, asumimos la necesidad de reflexionar sobre las instituciones de educación superior en cuanto espacio productor del pensamiento científico así como también por su rol en la construcción de relaciones sociales vinculadas a dicha producción de conocimiento en un contexto de diversidad cultural.

\section{El espacio académico y las nuevas configuraciones sociales}

En su tesis sobre la "invención de la Argentina" Nicolás Shumway (1993) sostiene que la estructuración de la argentinidad estaba basada en "ficciones orientadoras" que organizaron dicho proceso, en el cual la educación jugaba un rol central como generador de la movilidad social ascendente y el progreso económico. Sin embargo, el escenario histórico y social del que hiciéramos referencia más arriba, ha puesto de relevancia una transformación profunda que forzadamente debía impactar en esos modelos educativos y sociales. Lo que se observa es un cambio en el patrón de la demanda social que se manifiesta claramente en las nuevas configuraciones sociales; el sistema educativo en general y la universidad en particular, no podían dejar de reflejarlo convirtiéndose incluso, en uno de los espacios más complejos y enriquecidos en cuanto a las manifestaciones de dichas configuraciones. "En los últimos años, los profundos cambios sociales, políticos y económicos ocurridos en el país han establecido la necesidad, en las universidades, de adecuarse a un nuevo contexto determinando un proceso de transformación de éstas tanto al interior de sus estructuras como hacia el exterior, es decir, en sus relaciones con el Estado y con las demás instituciones de la sociedad" (Pochulu, 2004: 1). Las universidades experimentan un interesante y masivo proceso de matriculación de jóvenes procedentes de diferentes regiones, países y culturas que, por variadas razones, se establecen en nuestro país con el objetivo de realizar en él su formación profesional. Esa situación es en sí misma promotora de un análisis particular que debe insertarse en el contexto general de la globalización y el cambio en el mapa socio-cultural.

¿Cómo se manifiestan esas nuevas configuraciones sociales en el espacio académico? ¿Qué particularidades alcanzan en las actividades cotidianas en el aula? Estos interrogantes podrían dar paso a un 
análisis de especificidad pedagógica en el sentido de establecer estrategias de enseñanza-aprendizaje que permitan una mejor articulación entre los sujetos y los conocimientos, en busca de alcanzar mejores rendimientos en el proceso educativo. Sin embargo creemos que estas posibles y necesarias búsquedas didácticas deberían primero fundarse en un marco de conceptualización, de interpretación de la problemática en la que se tendrían que insertar. Los espacios académicos y las aulas como unidades de representación de los mismos, sufren un permanente proceso de cambio y transformación como consecuencia de los cambios y las transformaciones que vivencia la sociedad en su conjunto. Resultado de ello son las "nuevas demandas" que las generaciones de jóvenes e incluso el mercado laboral efectúan sobre los sectores responsables de organizar y llevar adelante el sistema educativo. Una de esas situaciones diferenciales es la conformación de espacios educativos pluriculturales.

La existencia en las aulas de diversos sujetos pertenecientes a diferentes "grupos culturales" no representa en sí misma una novedad; por el contrario, si asumimos que la tan mentada homogeneidad cultural pretendida por los forjadores del proyecto nacional del que hiciéramos mención más arriba, es exclusivamente una "ficción orientadora" o incluso un andamiaje simbólico de construcción social y política, comprenderemos que la sociedad argentina (y en extensión podríamos llevar este postulado al resto de las sociedades) no fue ni es en el sentido literal del término, homogénea. Lo que marca la diferencia, el cambio respecto de este paradigma socio-educativo, es el reconocimiento de esas diversidades culturales como poseedoras de derecho, como representantes de culturas en equidad, como portadoras de otros universos de sentido que, como mínimo, producen cambios en las perspectivas de abordaje. "La propia pluralidad de culturas contribuye a la diversidad de paradigmas científicos, en tanto condiciona la producción del saber y presenta objetos de conocimiento con configuraciones muy variadas" (García Canclini, 2004: 30).

Uno de los ejes centrales de esta nueva configuración del escenario educativo es el de la construcción de canales de comunicación entre los diferentes actores involucrados: nos referimos a una comunicación dialógica en términos bajtianos, es decir, una comunicación con horizontalidad de sentidos que, sin dejar de comprender la existencia de roles no asuma éstos como marcadores de valor en cuanto a la producción de diferencias jerárquicas. ¿Cómo se transita este proceso de apertura cognitiva hacia la diversidad? Sin mérito de terminar reduciendo nuestra propuesta a un "breve pero exhaustivo listado" de pasos a seguir, queremos primero destacar que un aspecto crucial en la construcción de una nueva perspectiva de abordaje del espacio académico es la de interrogarnos respecto del lugar del observador, es decir, de cómo conocemos y entendemos estas nuevas configuraciones sociales que tienen representación en nuestras aulas, de las que se desprenden nuestras prácticas educativas cotidianas. Creemos que uno de los caminos más significativos puede encontrarse en la creación de espacios reflexivos de intercambio y formación de aquellos que están involucrados con los niveles decisorios institucionales y con los profesionales dedicados a las tareas de enseñanza.

En su investigación sobre la relación entre prácticas pedagógicas, aspectos curriculares y equipos docentes desarrollada en el ámbito de la Universidad Nacional del Nordeste, Mariana Cecilia Ojeda y María Teresa Alcalá plantean que "los profesores, al desarrollar las prácticas de enseñanza, conforman ciertos repertorios que les permiten interactuar con los estudiantes y configurar de cierto modo los ambientes destinados a la educación, esto posibilita identificar ciertas prácticas constantes en el desarrollo del proceso que van generando modalidades de enseñanza”. En relación a estas modalidades, sostienen la importancia de promover interacciones personales generando ambientes que favorezcan la "construcción en el aula de grupos o comunidades de aprendizaje con la intención que los alumnos desarrollen relaciones cooperativas para lograr los aprendizajes" (2004: 2-3). La 
puesta en práctica de nuevas modalidades áulicas implica el abandono de metodologías centradas en la verticalidad de los vínculos cuyo eje se centra en acciones de transmisión unidireccional del conocimiento. Es en este sentido que sostenemos la importancia de transitar hacia perspectivas dialógicas de la educación, en las que se den paso a nuevas estrategias que se orienten hacia una transformación conceptual en cuanto a la producción social del conocimiento.

\section{Ciencia y diversidad}

"Investigar significa indagar sobre la realidad. Es un camino que nos conduce a responder a nuestros interrogantes sobre el porqué, el cómo y el para qué de los hechos. Estos interrogantes sobre la realidad surgen inicialmente a partir de una situación problemática" (Fassio y otros, 2006: 17). La investigación científica como tal ha estado, en distintos momentos históricos, en el centro de importantes debates ya sea de carácter epistémico, como también en el orden político, social y económico. Algunos de los ejes de discusión giran en torno a la categorización de las ciencias según su objeto de estudio y método, y otros ponen el foco en un aspecto sumamente interesante como lo es si el pensamiento científico puede construirse al margen del devenir de la sociedad que los produce. Aunque existen importantes "especialistas" de cada especificidad de dicha problemática, nos aventuramos a afirmar que cada uno de estos aspectos del problema son esencialmente eso: aspectos de una problemática única cuyo centro gira puntualmente en torno a quiénes producen el conocimiento y para qué lo hacen. Es decir, qué relaciones sociales y políticas sostienen la construcción del saber científico.

Como ya lo mencionáramos anteriormente, la Universidad fue y continua siendo la institución por excelencia cuyo rol central lo constituye la formación de los investigadores y la producción de innumerables proyectos de estudio científico. Si bien los cambios histórico-sociales han introducido modificaciones principalmente relacionadas con su carácter monopólico del conocimiento, está claro que la esencia de su función no ha sido alterada.

La pregunta que nos hacemos es ¿cómo se relacionan la construcción del conocimiento científico con los nuevos escenarios de diversidad que caracterizan nuestras aulas universitarias?

Uno de los puntos destacables de estos "nuevos escenarios" que involucran las posibles nuevas perspectivas globales, es una especie de renacimiento del sujeto como protagonista social indiscutido. Sería necio de nuestra parte negar el lugar protagónico del sujeto en la revolución del conocimiento surgida hace ya cientos de años: nos referimos puntualmente a un nuevo sujeto (usamos esta expresión sin otras intenciones que las expresadas) en el sentido de la existencia de las nuevas configuraciones sociales que lo constituyen. Un sujeto diverso, pluricultural y global que inserto en el espacio académico, busca que éste lo represente y lo potencie en términos diferenciadores de sus perspectivas como sujeto. La persistencia y cristalización de unas acciones unilaterales y por ende a-históricas, en relación a la conceptualización de la educación, la configuración de los métodos científicos y pedagógicos, e incluso de los roles y funciones de docentes y alumnos en el proceso educativo, ubica el escenario académico en un "callejón sin salida". Las acciones de los nuevos sujetos pedagógicos no pueden ni deben quedar aisladas de las cambiantes perspectivas socio-económicas.

No queremos decir con ello que compartimos ciertas tendencias internacionales que se han instalado en nuestra región, respecto del abandono, la renuncia e incluso llegando mucho más lejos, el total repudio de las prácticas y modelos que hasta la fecha le han significado a nuestras sociedades inconmensurables aportes. Lo que queremos destacar una y otra vez, es que, así como los proyectos homogeneizantes respondieron a un pensamiento y modelos de organización político, económico 
y social, el presente debe o se debe el debate, la construcción de los espacios que nos posibiliten comenzar a definir qué prácticas y qué modelos sumamos a las experiencias educativas para que estos nuevos sujetos, nosotros mismos, articulen prácticas democratizadoras de las nuevas configuraciones sociales. Lejos de adherir a la creación de propuestas totalizadoras que no hagan más que reemplazar un esquema por otro igual de homogéneo y totalizador, suscribimos la imperiosa necesidad de establecer los canales comunicacionales que dicha tarea requieren, transformando el aula universitaria en espacios de diálogo multidireccionales en los que todos los sujetos en su condición de individuos puedan constituirse en sujetos sociales dinámicos y aportantes. Como expresa Samaja (2003) no se trata de enarbolar el vínculo universidad-ciencia-desarrollo económico competitivo en reemplazo del proyecto de progreso-sociedad homogénea, sino todo lo contrario. Para el autor, la búsqueda de hoy, el desafío actual consiste en asumir construcciones académicas que integren lo tecnológico del presente con lo experimental, reflexivo, tradicional y perceptual del pasado, desde una perspectiva renovadora y, agregamos nosotros, pluricultural del conocimiento y la cultura.

\section{Conclusiones}

Pensar la Universidad en la Argentina actual es en sí mismo todo un desafío. Pensar el espacio académico dentro de ese contexto y problematizarlo en el marco de un proceso de crisis y cambios no sólo en el plano local sino en lo global resulta mucho más complejo. Las prácticas sociales de debate que nos han caracterizado como sociedad durante un período extenso de nuestra historia reciente, se han abandonado progresivamente y en su reemplazo nos hemos inclinado, algunos por incesantes discusiones estériles de teorías "novedosas" o de formulismos de enunciación, otros por la búsqueda de recetas mágicas que nos dijeran cómo resolver unos problemas que a la postre, y a pesar de intentar su aplicación con una ceguera majestuosa, persisten.

Nos encontramos atravesando una encrucijada por demás compleja y novedosa que, además de presentar peculiaridades en los aspectos sociales, políticos o económicos, nos propone realizar una mirada crítica sobre los procesos formativos de nuestra sociedad así como también de las interrelaciones que los sujetos construyen tanto en el ámbito de la cultura como de la educación.

La tendencia a producir esquemas o modelos de interpretación cuyas aplicaciones deban seguirse al pie de la letra, incluso en aquellos casos cuyas elaboraciones no se hayan realizado tomando como referencia nuestras necesidades y perspectivas sociales, continúa vigente a pesar de los innumerables inconvenientes que resultan de ello.

Como expresamos a lo largo de este trabajo, la problemática de la que hacemos mención no es una novedad en el sentido estricto del término. Por el contrario, hablar de sociedades plurales, de culturas diversas, es una práctica que muchos sectores sociales vienen realizando hace ya largo tiempo; incluso, algunas corrientes de las distintas ciencias sociales han propiciado estudios asumiendo a la diversidad como un enunciado descriptivo o también como toda una corriente interpretativa.

Lo cierto es que, la globalización ha puesto de manifiesto unos flujos de intercomunicación que han acelerado procesos de transformación en todos los planos anteriormente mencionados. La educación superior y la producción del conocimiento, no quedan al margen de dicho fenómeno.

Por ello insistimos, lo nuevo, lo necesario, lo constructivo resulta de la generación de espacios dialógicos, de interrelaciones de horizontalidad que promuevan el desarrollo de unas teorías y unas prácticas que se orienten a la producción de proyectos múltiples, sin entender por ello que los mismos "tengan un poquito de cada cosa". Por el contrario, creemos que el gran desafío es superar 
esa instancia de "recorte y pegue" en los modelos académicos y las planificaciones pedagógicas en las que se asumen los "nuevos lenguajes" como formulismos de enunciación que lejos están siquiera de corresponderse a unas teorías de interpretación y acción.

Proyectar el espacio académico hacia una nueva perspectiva de acción, propiciar el diálogo continuo entre los diversos actores sociales e incluir lo diverso asumiendo las asimetrías de dicha diversidad, es por todo, la intención primera de este trabajo. El aula puede reescribirse en forma continua y generar esos ambientes en los que ciencia y pluralidad dialoguen renovadamente.

\section{Referencias Bibliográficas}

Cuche, Denys. (2004). La noción de la cultura en las ciencias sociales. Buenos Aires: Nueva Visión.

Devalle, Susana B. C. (1995). “Concepciones de la diferencia y naturaleza de la etnicidad” en: Murphy, Susana B. (comp.) El Otro en la Historia: el extranjero. Universidad de Buenos Aires.

Dussel, Inés. (2003). "La producción de la exclusión en el aula: una revisión de la escuela moderna en América Latina” Ponencia presentada en la X Jornada LOGSE, Granada en: Revista Papeles, 1, 1, Granada: Grupo de Investigación Políticas y Reformas Educativas, pp. 75-96.

Fassio, A. Pascual, L. y Suárez F.M. (2006). Introducción la Metodología de la Investigación. Aplicada al saber administrativo y al análisis organizacional. Buenos Aires: Ediciones Macchi.

Foucault, Michel. (1993). Las palabras y las cosas. México: Siglo XXI.

García Canclini, Néstor. (2004). Diferentes, desiguales y desconectados. Mapas de la Interculturalidad. Buenos Aires: Gedisa. Gómez Campo, V.M. y Tenti Fanfani, E. (1989). Universidad y Profesiones. Crisis y alternativas. Buenos Aires: Miño y Dávila Editores.

Hobsbawm, Eric (1995). Historia del Siglo XX. 1914-1991. España: Crítica.

León-Portilla, Miguel. (coord.) (2001). Motivos de la Antropología Americanista. Indagaciones en la diferencia. México: F.C.E. Ojeda, M. C. y Alcalá, M. T. (2004). La enseñanza en las aulas universitarias. Una mirada desde las cátedras: aspectos curriculares que inciden en las prácticas pedagógicas de los equipos docentes. Revista iberoamericana de Educación Disponible en: www.rieoei.org/deloslectores/765Ojeda.PDF

Pochulu, Marcel David. (2004). La Educación Superior Argentina hoy, entre instituciones que se transforman y relaciones que se modifican. Revista iberoamericana de Educación. Disponible en: www.rieoei.org/deloslectores/796Pochulu.PDF Samaja, Juan. (2003). Sobre la ciencia, la técnica y la sociedad. Para pensar la nueva agenda de la educación superior, en: Ciencia, Docencia y Tecnología, No 27, Año XIV, UNER.

Shumway, Nicolás. (1993). La invención de la Argentina. Historia de una idea. Buenos Aires, Emecé.

Todorov, Tzvetan. (1987). La Conquista de América. La cuestión del otro. México, SigloXXI.

Summary: Cultural diversity has become one of the axes of the academic and pedagogical process, as much as for the production of knowledge -able to give account of this phenomenon- than for strategies of pedagogical intervention that allow us to transport the educative processes with the expected success. In this complex and symbolic multiplicity of the classroom spaces, "the well-known" is enhanced defying us in the continuous search of new proposals and strategies that, in saying of Garcia Canclini (2004), orient us with respect to "where is possible to find the theory that organizes ourselves on the new diversities". Globalisation has become the present organizer of social processes of sensible mobility and, at the same time, in a relational perspective producer that is constituted in a cognitive challenge for education. This work aims to reflect on these social and educative processes, assuming that the role of the academic space and therefore of the scientific 
thought are distinctive in the construction of intercultural societies in which the problematic "of cultural diversity" finds ways of to enhance itself in new motivating knowledge of democratic thought.

Key words: academic space - cultural diversity - pedagogical intervention - scientific thought.

Resumo: A problemática da diversidade cultural transformou-se num dos eixos do processo acadêmico e pedagógico, tanto no que refere-se à produção de saberes capazes de dar conta de dito fenómeno, como de estratégias de intervenção pedagógica que nos permitam conduzir os processos educativos com o sucesso esperado. Neste contexto de complexidade e multiplicidade simbólica dos espaços áulicos, representam-se incomensuráveis unidades de sentido que potencian "o conhecido" e nos desafiam na busca contínua de novas propostas e estratégias de ação que, conforme García Canclini (2004) orientem-nos respecto de onde "encontrar a teoria que organize as novas diversidades".

A globalização mundial constituiu-se num presente organizador de processos sociais de sensível movimento e, ao tempo, em produtor de perspectivas relacionais que em se mesmas constituem-se num desafio cognitivo no que a educação se refere. O presente trabalho se propõe reflexionar sobre estes processos sociais e educativos, assumindo que o papel do espaço acadêmico e portanto do pensamento científico, resultam definitórios na construção de sociedades interculturais nas que a mencionada "problemática da diversidade cultural" encontre formas de potenciar-se em novos conhecimentos motivadores do pensamento democrático.

Palavras chave: diversidade cultural - espaço acadêmico - intervenção pedagógica - pensamento científico.

${ }^{(*)}$ Profesora de Historia (Universidad Católica de Salta / INSP “Dr.J.V.G.”). Profesora de la Universidad de Palermo en el Departamento de Publicidad de la Facultad de Diseño y Comunicación. Integra equipo bi-nacional de investigación en ciencias sociales. Especialista en Políticas Educativas del Estado sobre Poblaciones Aborígenes Americanas. 